\title{
Enterococcus faecium Alleviates Gut Barrier Injury in C57BL/6 Mice with Dextran Sulfate Sodium-Induced Ulcerative Colitis
}

\author{
Wei He, ${ }^{1}$ Weijie $\mathrm{Ni}^{2}$ and Junning Zhao $\mathbb{D}^{1}$ \\ ${ }^{1}$ Department of Gastroenterology, Geriatric Hospital of Nanjing Medical University, Nanjing 210024, China \\ ${ }^{2}$ School of Medicine, Southeast University, Nanjing 210009, China \\ Correspondence should be addressed to Junning Zhao; zj11102@163.com
}

Received 5 June 2020; Revised 27 October 2021; Accepted 2 November 2021; Published 25 November 2021

Academic Editor: Amosy M'Koma

Copyright ( 2021 Wei He et al. This is an open access article distributed under the Creative Commons Attribution License, which permits unrestricted use, distribution, and reproduction in any medium, provided the original work is properly cited.

\begin{abstract}
The involvement of gut microbiota composition in ulcerative colitis is strongly supported by previous research. Growing evidence suggests that probiotic therapy protects against inflammatory bowel disease in animal models and patients. However, as a probiotic, the role of Enterococcus faecium (E. faecium) in UC remains unclear. Nevertheless, the potential mechanism of the protective effect of E. faecium remains unknown. In this study, a dextran sulphate sodium-induced (DSS-induced) colitis model was used to detect the underlying mechanism of E. faecium in maintaining gut homeostasis. ELISA was performed to detect the levels of cytokines (TNF- $\alpha$, IL-1 $\beta$, IL-6, and IL-10). Furthermore, 454 pyrosequencing was used to investigate the microbiota composition in fecal samples. The results illustrate that E. faecium administration could prevent DSS-induced gut inflammation and intestinal flora imbalance. At the same time, the damage to intestinal mucosal barrier and tight junctions was partially repaired. These results demonstrate the preventive effect of E. faecium in DSS-induced intestinal injury. The present study provides new insights into the medicinal value of E. faecium for UC.
\end{abstract}

\section{Introduction}

Inflammatory bowel disease (IBD) is complex and presents many challenges to gastroenterologists [1]. Ulcerative colitis (UC) is a major form of IBD, which is marked by aberrant inflammation within the human colon [2]. Previous studies suggest that microbiota dysbiosis and intestinal barrier dysfunction are closely related to UC [3].

Although there have been many studies on UC, its pathological mechanism remains poorly understood [4]. Previous research has demonstrated that epithelial barrier and mucous barrier defects are strongly related to the pathogenesis of UC [5]. Furthermore, Suzuki indicated that the expression of occludin and zonula occludens-1 (ZO-1), which are tight junction proteins, is associated with gut permeability [6]. Ma et al. confirmed that the expression level of ZO-1 is negatively correlated with the degree of UC [7]. Recent studies have shown that microbiota could have an impact on intestinal permeability [8].

The intestinal microbiota, which seems to be a hidden organ composed of trillions of microorganisms, makes essential contributions to intestinal homeostasis [9]. Probiotics are defined as nonpathogenic living microorganisms that are beneficial to disease treatment or host health. A series of studies confirmed that imbalance of intestinal microflora refers to the pathogenesis of UC [3]. Enterococcus faecium (E. faecium) is a type of probiotic that inhabits the human gastrointestinal tract [10]. A previous study showed that regular intake of Enterococcus faecium CRL 183 could reduce the DSS-induced colitis injury on rats $[11,12]$. Consistent with the effect in Caenorhabditis elegans, Pedicord et al. observed that E. faecium colonization improves host intestinal epithelial defense programs in mice [13]. Increasing evidence has demonstrated that E. faecium is associated with gut protection. In this study, the relationship between E. faecium administration and gut barrier integrity was analyzed.

The protective mechanism of probiotics against UC is multifaceted. The inhibition of intestinal pathogenic bacteria by probiotics plays a crucial role in the protective effect of probiotics in UC. Although previous studies have illustrated that E. faecium could have gut-protective effects $[11,12]$, 
there are still studies showing controversial results [13]. It is of great value to explore the mechanism of E. faecium in UC.

In this work, we investigated the role of E. faecium on dextran sulphate sodium-induced (DSS-induced) intestinal injury in order to provide new methods for UC treatment.

\section{Materials and Methods}

2.1. Animals. Male C57BL/6 mice (body weight, 22 to $24 \mathrm{~g}$; 7-8 weeks old) were purchased from Shanghai Jiesijie Experimental Animal Co., Ltd. (Pujiang, Shanghai, China). Before the experiment, animals were adaptively fed for a week. During the experiment, the mice were weighed daily to observe the changes in body weight. All mice were maintained under standard conditions in an animal house at Nanjing Medical University. Experimental ulcerative colitis was induced by dextrose sulfate sodium (DSS, 3\% wt/vol, dissolved in $\mathrm{ddH} 2 \mathrm{O}$, administered from day 1 to day 7). Mice were randomly chosen to receive different treatments via gavage from day 1 to day 7, including E. faecium (R026, Beijing Hanmi Pharm Co., Ltd., Beijing, China, $1 \times 10^{9} \mathrm{CFU} /$ day, $n=16$ ) and phosphate-buffered saline (PBS) $(n=16)$. On the eighth day, the bleeding scores and colon length were assessed. Half of the mice were sacrificed on day 8 , and the other mice were sacrificed on day 14 to observe the effect of E. faecium on survival. All animal studies were approved by the Institutional Animal Care and Use Committee of Nanjing Medical University.

2.2. Stool Consistency and Bleeding Score. Stool consistency was assigned grades according to the following criteria: normal stool consistency with negative hemoccult: 0 , soft stools with positive hemoccult: 1, very soft stools with traces of blood: 2, and watery stools with visible rectal bleeding: 3 .

Bleeding score was assigned grades according to the following criteria: normal stool consistency with negative hemoccult: 0 , soft stools without positive hemoccult: 1 , soft stools with positive hemoccult: 2 , very soft stools with traces of blood: 3, and watery stools with visible rectal bleeding: 4 .

2.3. Colon Pathology and Immunohistochemistry. Colons from mice were excised, flushed, and collected. Colon tissue was fixed in Bouin's fluid (Sigma-Aldrich) and embedded in paraffin. Five micrometer sections of paraffin-embedded colons were prepared for further experiments. Alcian blue staining and HE staining were used to examine mucosal damage. For immunofluorescence (IF), the sections were incubated with anti-ZO-1 antibody (1:200 Abcam; Cambridge, MA) and anti-claudin antibody (1:200 Abcam; Cambridge, MA) at $4^{\circ} \mathrm{C}$. All the results were observed by microscopy. The above histological analysis was performed in a blinded manner to avoid bias.

2.4. Intestinal Permeability. Disaccharide permeability probes $(50 \mathrm{mg} / \mathrm{ml}$ mannitol and $100 \mathrm{mg} / \mathrm{ml}$ lactulose) were used to detect intestinal permeability. DSS-treated mice administered phosphate-buffered saline or E. faecium ( $n=4$; 4 days) were given a probe after fasting for $12 \mathrm{~h}$ by oral gavage, and urine was collected after $12 \mathrm{~h}$. Highperformance liquid chromatography was performed to mea- sure the urine concentrations of lactulose and mannitol. The recovery rates of lactulose and mannitol were examined to evaluate intestinal permeability.

2.5. Short-Chain Fatty Acid (SCFA) Assay. Fecal samples were collected from the metabolism cage on the 8th day after DSS treatment. After mixing with distilled water, the fecal samples were centrifuged $(2,500 \mathrm{xg})$. The samples were then extracted with ether and sulfuric acid. The ether phase was collected and used to examine the total SCFA concentrations by an Agilent $6890 \mathrm{~N}$ gas chromatography machine (Agilent Technologies CA USA).

2.6. Serum LPS and Cytokines. Blood samples were collected from the left ventricle on the 8th day after DSS treatment. A protein-based enzyme-linked immunosorbent assay (Elisa) was used to detect the levels of cytokines (RAB0274; RAB0477; RAB0308; RAB0245, Merck, USA). In brief, after the antibody was coincubated overnight with the plate, the sample to be tested was added, followed by a reaction solution for coloration, and the level of cytokine was detected by colorimetry. Limulus test kit (EC644405; Xiamen Houshiji, Ltd., Xiamen, China) was used to detect LPS.

2.7. Microbiological Analysis of Fecal Samples. 454 pyrosequencing was used to determine the microbiota composition by targeting the V3-V4 region of the bacterial $16 \mathrm{~S}$ rRNA gene. Sequences were binned for a minimal sequence length of $300 \mathrm{bp}$, a minimal base quality threshold of 30 cycles, and a maximum homopolymer length of 6 cycles. Sequences were further clustered into operational taxonomic units (OTUs) or phylotypes. According to the results, Seqmatch and Blastall were used to assign the closest taxonomic neighbors and relative bacterial species.

2.8. Statistical Analysis. Data are presented as the mean \pm SD . Between-group comparisons were performed by one-way ANOVA followed by Dunnett's multiple comparison test or Student's $t$-test. Differences with $P<0.05$ were considered statistically significant.

\section{Results}

3.1. E. Faecium Treatment Reduced Lethality and Alleviated Intestinal Injuries. DSS-mediated colitis was used to detect the mechanisms of E. faecium in protecting the intestine from injury. As shown in Figure 1(a), treatment with E. faecium reduced the lethality rate remarkably compared with the DSS + PBS group. Consistent with the survival curve, mice treated with E. faecium lost significantly less body weight after DSS treatment (Figure 1(b)). To further detect intestinal injuries in mice, we examined the intestinal tracts and anuses among the three groups. As shown in Figures 1(c) and 1(e), compared with the control group, the bleeding and anabrosis in the anus and intestinal tract of mice were alleviated by E. faecium treatment. Meanwhile, DSS induced colon necrosis and shorter colons, which were restored by E. faecium treatment (Figures 1(d) and 1(f)). Evaluation of stool consistency also demonstrated the therapeutic effect of E. faecium (Figure $1(\mathrm{~g})$ ). These results 


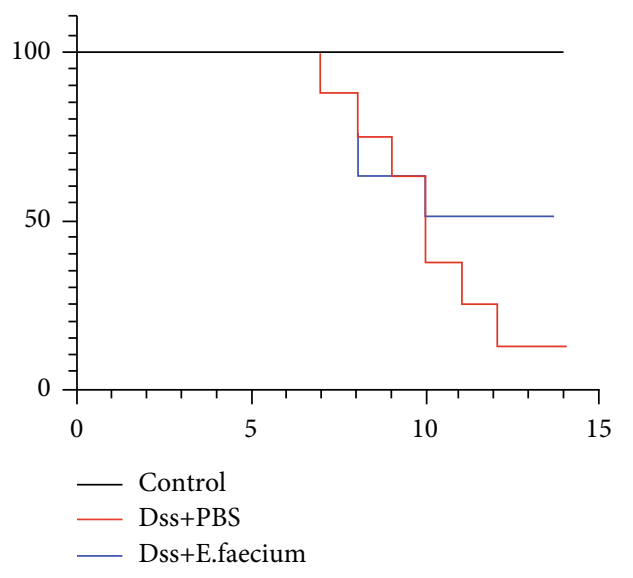

(a)

DSS+PBS
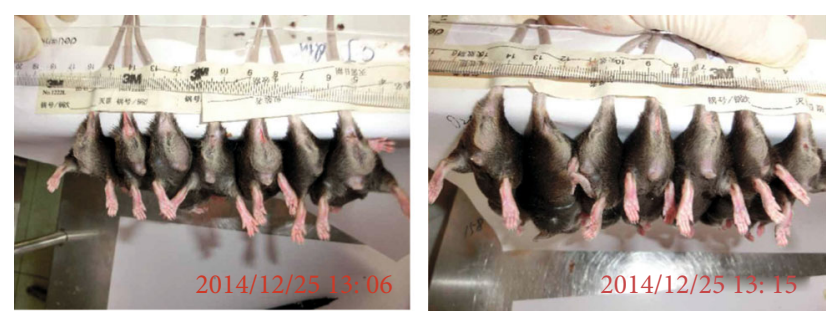

(c)

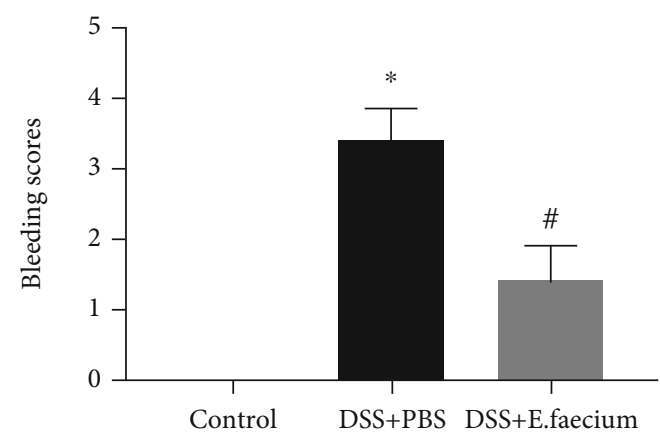

(e)

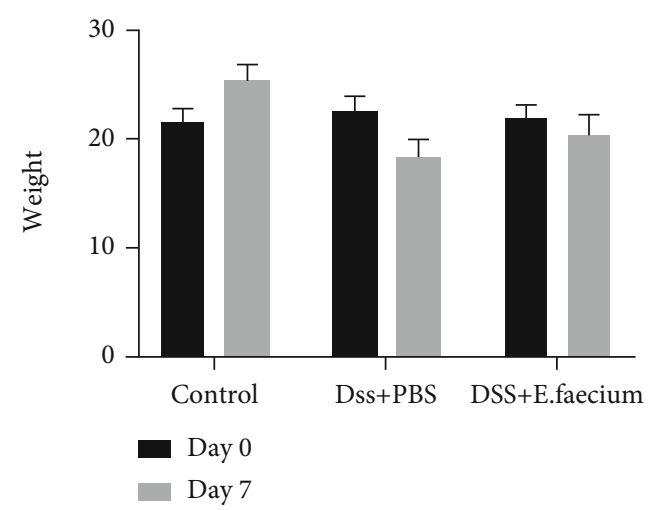

(b)

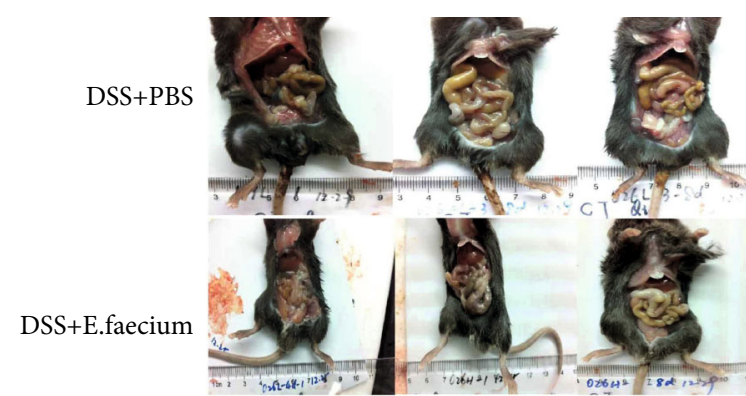

(d)

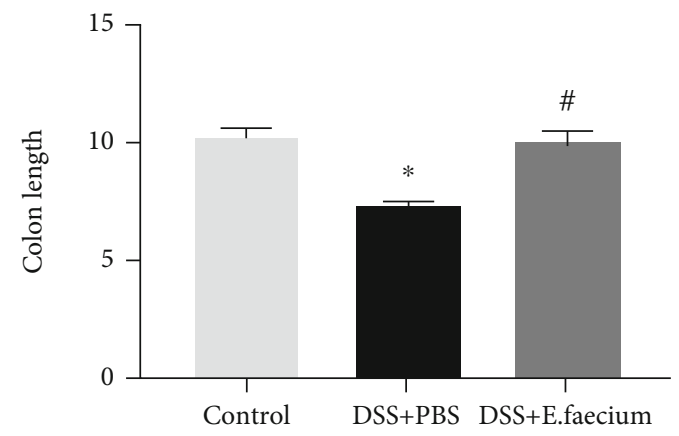

(f)

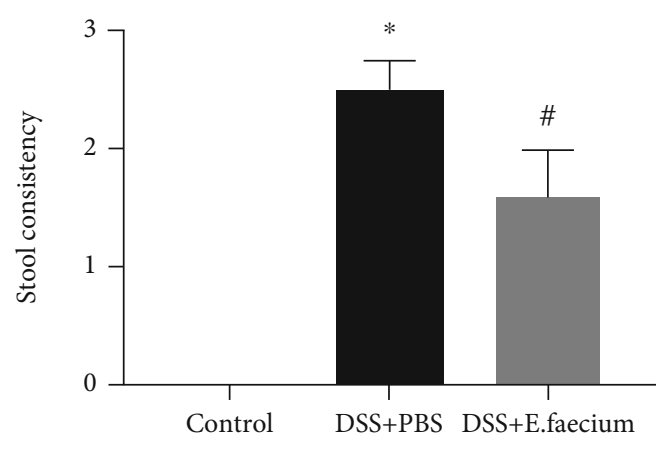

(g)

FIGURE 1: The protective effect of E. faecium against DSS-induced colitis. (a) The survival curve for DSS-induced colitis. (b) Weight loss. (c) Typical image of gut damage. (d) Typical image of colon length. (e) Bleeding scores. (f) Colon length. (g) Stool consistency. ${ }^{*} P<0.05$ vs. control group; $\# P<0.05$ vs. DSS + PBS group. 


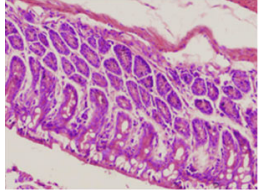

Control

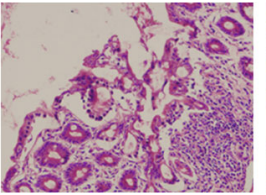

DSS+PBS

(a)

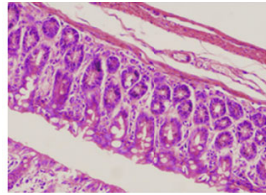

DSS+E.faecium

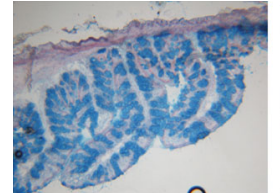

Control

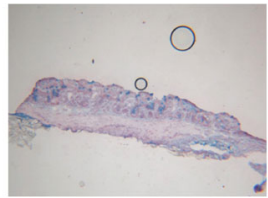

DSS+PBS

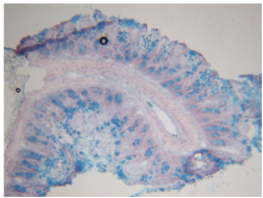

DSS+E.faecium

(b)

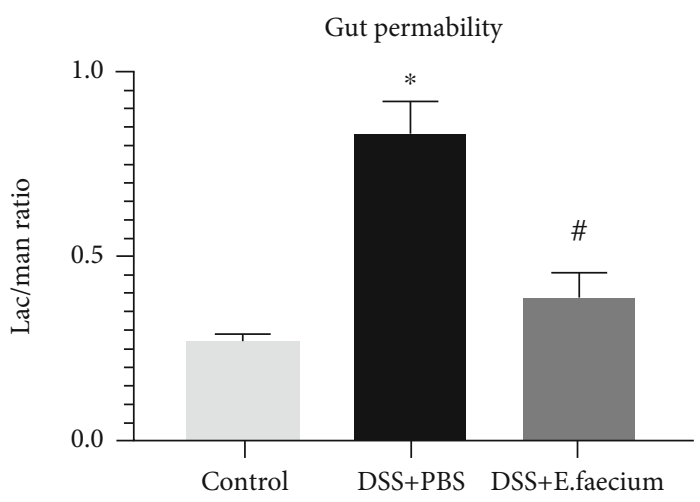

(c)

FIGURE 2: E. faecium restores intestinal mucosal damage induced by DSS. (a) HE staining of the colon. (b) Alcian blue staining of the colon. (c) Detection of intestinal permeability. ${ }^{*} P<0.05$ vs. control group; $\# P<0.05$ vs. DSS + PBS group.
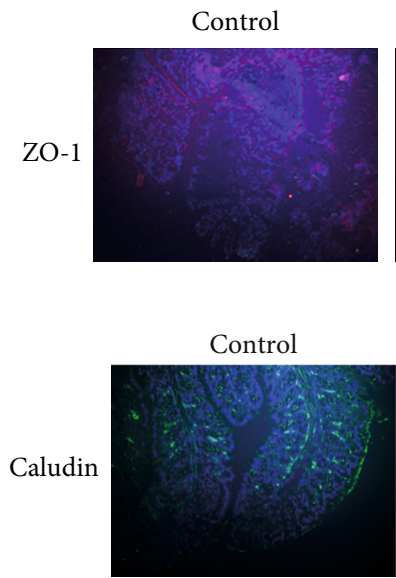

DSS+PBS

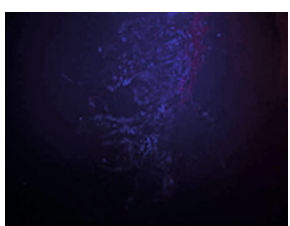

(a)

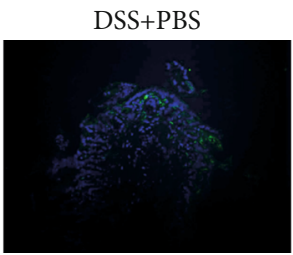

(b)
DSS+E.faecium

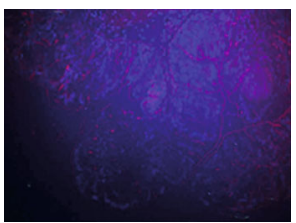

DSS+E.faecium

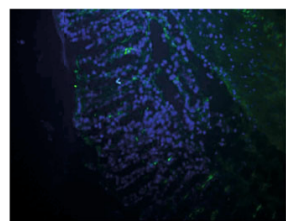

FiguRE 3: E. faecium protects against intestinal injury by strengthening tight junctions. Immunohistochemical staining of (a) ZO-1 and (b) claudin in the colon.

indicate that E. faecium administration prevents DDSinduced colon damage in vivo.

\subsection{E. Faecium Treatment Alleviated DSS-Induced Intestinal} Mucosal Damage. As the above research shows, E. faecium may protect against DSS-mediated colitis. Previous studies have shown that intestinal mucosal damage is an important phenotype in DSS-induced intestinal injury. To investigate the intestinal mucosal after E. faecium treatment, we performed HE staining and alcian blue staining. As shown in Figures 2(a) and 2(b), compared with the control group, inflammatory cell infiltration increased significantly after DSS treatment, and this change was reversed by E. faecium. In addition, the levels of mucins were upregulated in the $E$. faecium-treated group. Furthermore, intestinal permeability was measured to test the effect of E. faecium administration on DSS-induced intestinal mucosa damage. As shown in Figure 2(c), compared with the DSS + PBS group, the injury of intestinal permeability was alleviated by E. faecium treatment. These results suggest that treatment with E. faecium may prevent intestinal mucosa damage.

3.3. E. Faecium Treatment Helps to Restore Tight Junctions. To further explore the effect of E. faecium on the repair of tight junctions, we performed immunofluorescence to detect the expression and distribution of ZO-1 and claudins, which are tight junction-associated markers. As shown in Figure 3, ZO- 1 and claudins increased after E. faecium treatment. This 


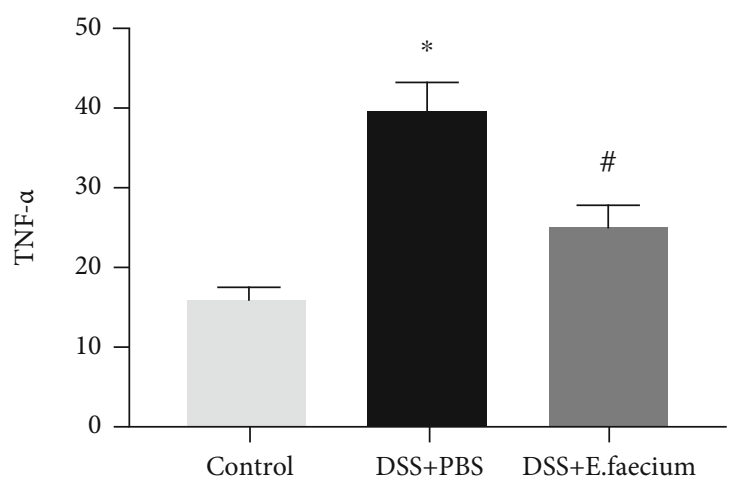

(a)

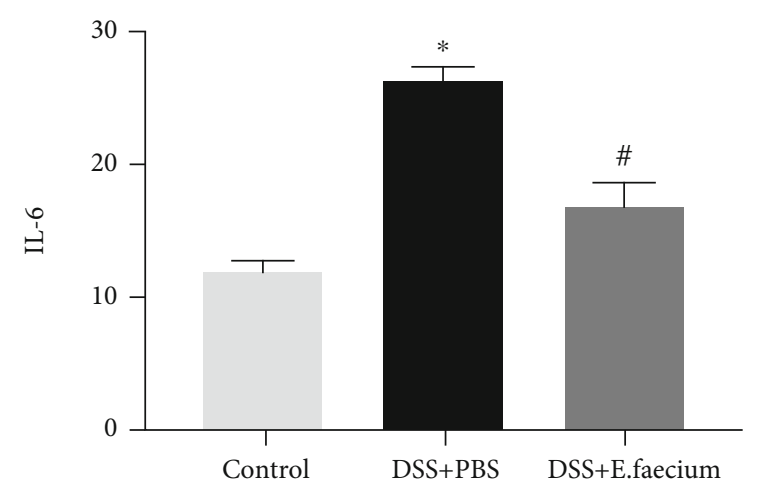

(c)

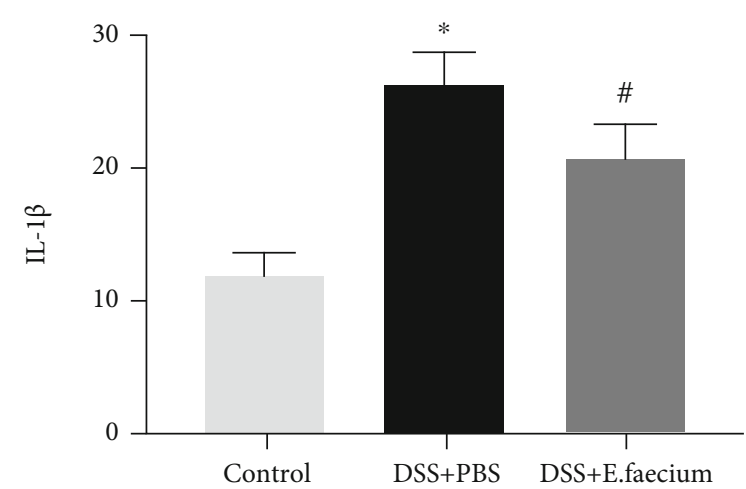

(b)

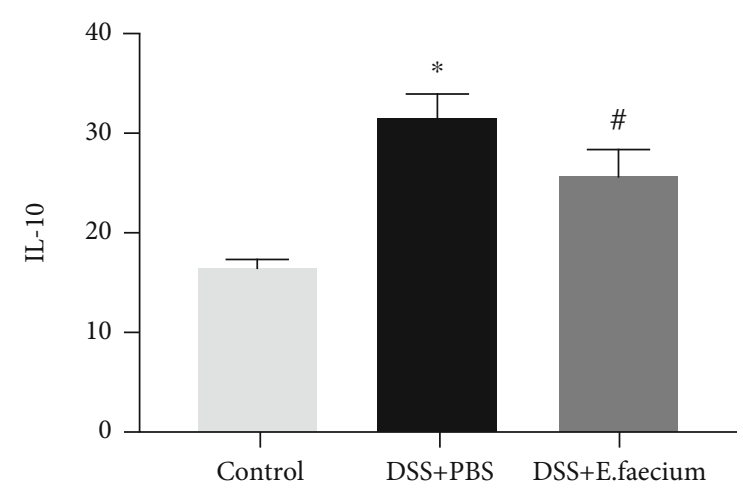

(d)

Figure 4: E. faecium treatment alleviates gut inflammation. Inflammatory cytokines: (a) TNF- $\alpha$, (b) IL- $1 \beta$, (c) IL-6, and (d) IL-10. * $P<0.05$ vs. control group; $\# P<0.05$ vs. DSS + PBS group.

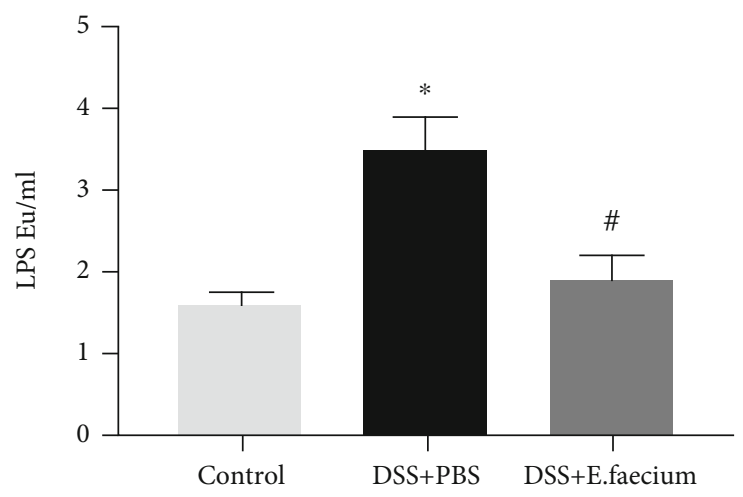

(a)

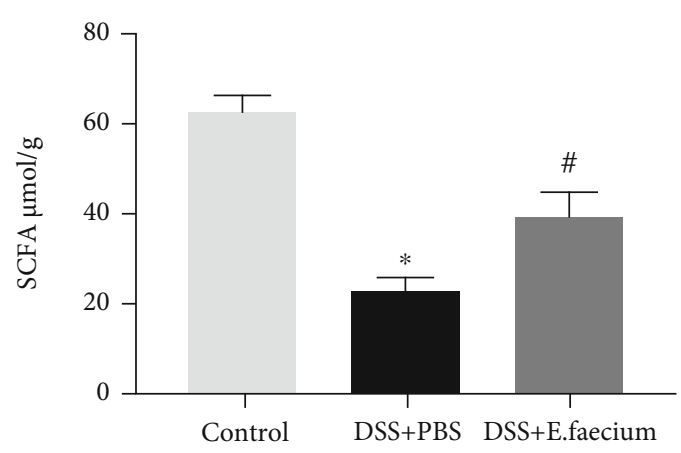

(b)

FIgURE 5: E. faecium treatment shifts the balance of inflammatory factors. The levels of (a) LPS and (b) SCFAs. ${ }^{*} P<0.05$ vs. control group; $\# P<0.05$ vs. DSS + PBS group.

result indicated that $E$. faecium was involved in the repair of the tight junction complex.

\subsection{E. Faecium Treatment Protected against DSS-Induced} Systemic Inflammation. A previous study demonstrated that the relief of inflammation is related to intestinal permeability. Therefore, we tested serum samples from mice by ELISA to assess the protective effect of E. faecium treatment on gut inflammation. Compared with the control group, the levels of plasma cytokines, including TNF- $\alpha$, IL-1 $\beta$, IL-6, and IL-
10, were significantly decreased in the E. faecium-treated group (Figure 4).

3.5. E. Faecium Treatment Regulated Anti- and Proinflammatory Factors. To further identify the potential mechanism of E. faecium in DSS-induced intestinal inflammation, the plasma LPS of mice was analyzed. As shown in Figure 5(a), the induction of LPS by DSS was reversed by E. faecium treatment. Furthermore, compared with the control group, the concentration of total SCFAs was significantly upregulated after E. faecium treatment (Figure 5(b)). 


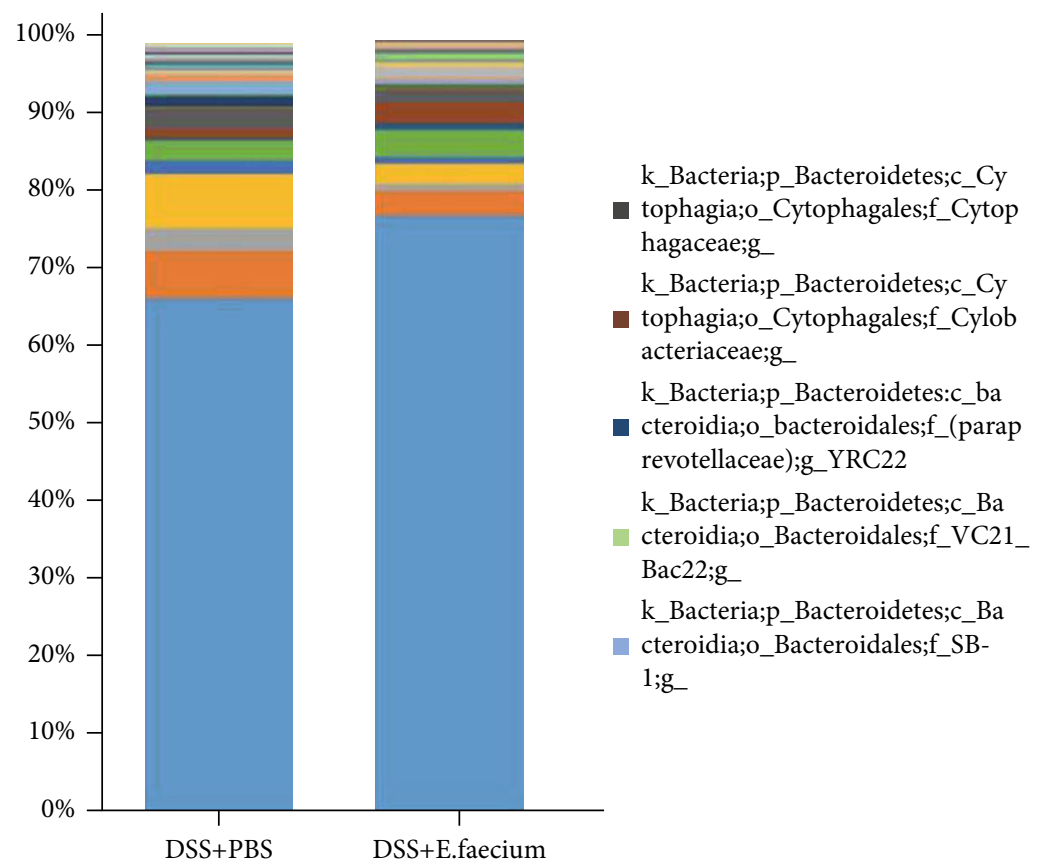

(a)

Figure 6: Continued. 


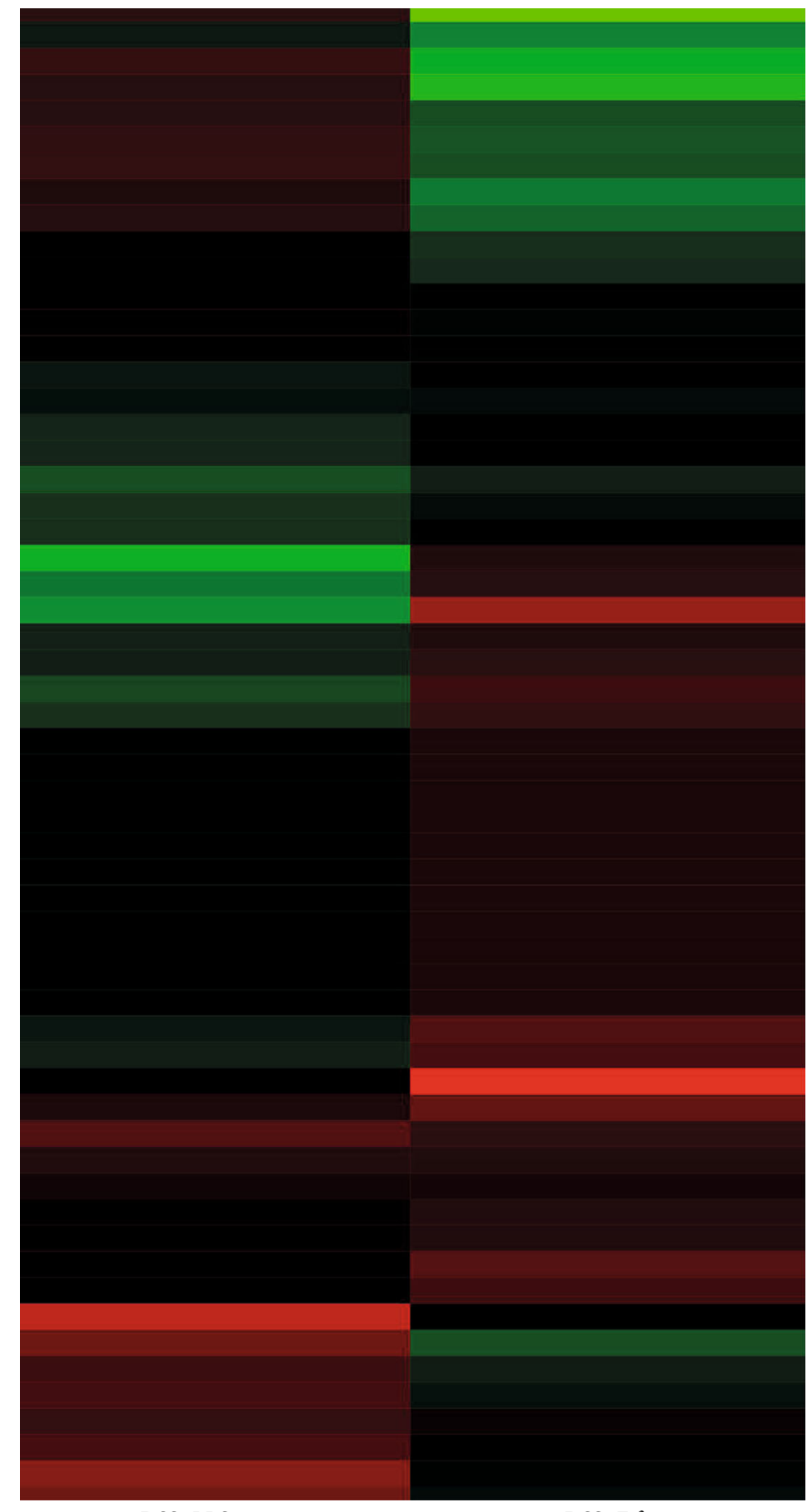

DSS+PBS
DSS+E.faecium

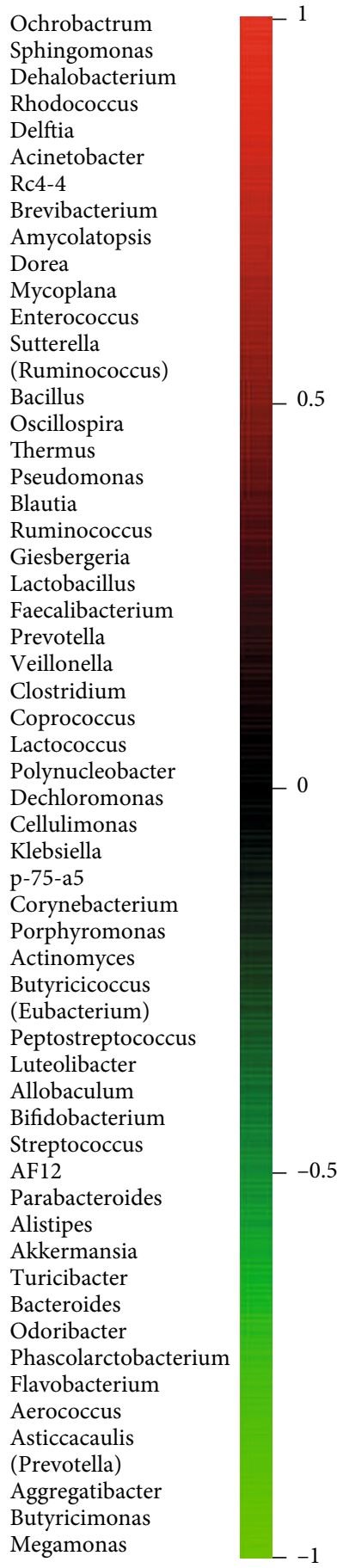

(b)

Figure 6: E. faecium treatment restores the imbalance of intestinal flora. (a) Microbiota composition in mice. (b) Heatmap of the abundance of bacterial genera in mice.

3.6. E. Faecium Treatment Restored the Imbalance of Intestinal Flora Induced by DSS. Trillions of microorganisms colonize the human gut. These microorganisms are related to regulating the immune system and balancing the internal environment. Therefore, in this study, we performed $16 \mathrm{~S}$ rDNA sequencing analysis to detect changes in the microbi- ota after E. faecium treatment. As shown in Figure 6(b), compared with the control group, the gut microbiota was significantly altered after E. faecium treatment. Specifically, the proportion of Ochrobactrum sp. and Acinetobacter sp. was reduced upon E. faecium treatment. Meanwhile, the proportion of Butyricicoccus sp. Lactobacillus sp., and 

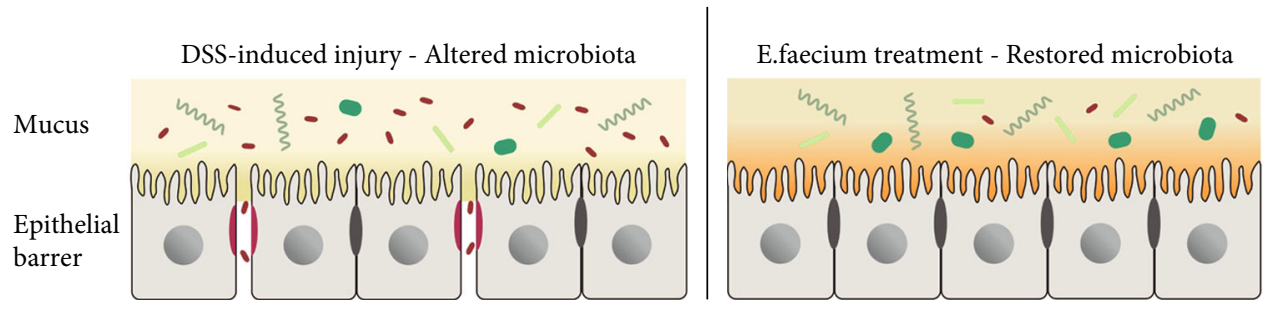

FIGURE 7: Working model of E. faecium treatment. E. faecium treatment improved the intestinal flora, thereby protecting the intestinal mucosa and reducing the damage to tight junctions.

Bifidobacterium sp. was upregulated. These results revealed the pivotal role of $E$. faecium in restoring the imbalance of intestinal flora induced by DSS.

\section{Discussion}

In this study, the effect of E. faecium treatment on a DSSinduced UC model was investigated. The remarkable finding of this study was that E. faecium markedly protected against intestinal damage induced by DSS and significantly decreased mortality. Based on alcian blue staining and gut permeability, E. faecium treatment contributed to intestinal mucosal injury. Meanwhile, the upregulation of ZO-1 and claudin confirmed that E. faecium was able to restore intestinal barrier function by increasing the concentration of tight junction proteins. Consistent with previous studies, $E$. faecium treatment reduced the intestinal damage induced by DSS, which further supports the hypothesis that E. faecium colonization improves host intestinal epithelial defense programs in rats [11]. Preview studies have demonstrated that the levels of TNF- $\alpha$, IL- $1 \beta$, IL-6, and IL-10 are upregulated in patients with IBD [14-16]. In the present study, our results also showed that the expression of TNF- $\alpha$, IL- $1 \beta$, IL6, and IL-10 was downregulated after E. faecium treatment.

Increasing evidence has confirmed the antiinflammatory functions of SCFAs. Previous studies have suggested that SCFA-producing bacteria are significantly downregulated in pediatric CD patients [16]. A study of metabolism and intestinal flora demonstrated that the reduction in SCFA-producing bacteria can result in metabolic stress in inflammatory disease [17]. E. faecium treatment increased the levels of SCFAs. Moreover, our data also showed that E. faecium treatment can restore the inhibition of Butyricicoccus sp., which contributes to butyrate generation and was decreased in IBD $[18,19]$.

Preview studies suggest that Ochrobactrum sp. and Acinetobacter sp. were increased in UC patients, while Lactobacillus sp., Butyricicoccus sp., and Bifidobacterium sp. were decreased [20-24]. The evidence presented so far implies that a balance of healthy microbiota is required to shape the immune system response during disease [25]. Meijer et al. indicated that beneficial species of bacteria, including Lactobacillus sp. and Bifidobacterium sp., can prevent or attenuate UC [26]. In this study, our results indicated that, compared with the control group, beneficial bacteria, including Lactobacillus sp., Butyricicoccus sp., and Bifidobacterium sp., were upregulated in the E. faecium-treated groups, demonstrating the potential therapeutic value of E. faecium.

In conclusion, in this study, it was confirmed that E. faecium administration prevents the intestinal mucosa and reduces damage to tight junctions, as summarized in Figure 7. E. faecium could reduce intestinal damage and improve the imbalance of the intestinal flora induced by DSS. This study explored the potential mechanisms of E. faecium treatment and provided new insight into the intervention and treatment of UC.

\section{Data Availability}

We declare that the data used to support the findings of this study are available from the corresponding author upon request.

\section{Disclosure}

The abstract was presented in "Wiley Online Library" [https://onlinelibrary.wiley.com/doi/10.1111/1751-

2980.12954].

\section{Conflicts of Interest}

All authors declare no conflict of interest.

\section{Authors' Contributions}

The manuscript has been read and approved by all the authors.

\section{References}

[1] R. J. Xavier and D. K. Podolsky, "Unravelling the pathogenesis of inflammatory bowel disease," Nature, vol. 448, no. 7152, pp. 427-434, 2007.

[2] R. Hodson, "Inflammatory bowel disease," Nature, vol. 540, no. 7634, p. S97, 2016.

[3] K. L. Glassner, B. P. Abraham, and E. Quigley, "The microbiome and inflammatory bowel disease," The Journal of Allergy and Clinical Immunology, vol. 145, no. 1, pp. 16-27, 2020.

[4] K. Conrad, D. Roggenbuck, and M. W. Laass, "Diagnosis and classification of ulcerative colitis," Autoimmunity Reviews, vol. 13, no. 4-5, pp. 463-466, 2014.

[5] R. Ungaro, S. Mehandru, P. B. Allen, L. Peyrin-Biroulet, and J. F. Colombel, "Ulcerative colitis," The Lancet, vol. 389, no. 10080, pp. 1756-1770, 2017. 
[6] T. Suzuki, "Regulation of the intestinal barrier by nutrients: the role of tight junctions," Animal Science Journal, vol. 91, no. 1, article e13357, 2020.

[7] T. M. Ma, N. Xu, X. D. Ma, Z. H. Bai, X. Tao, and H. C. Yan, "Moxibustion regulates inflammatory mediators and colonic mucosal barrier in ulcerative colitis rats," World Journal of Gastroenterology, vol. 22, no. 8, pp. 2566-2575, 2016.

[8] C. Graziani, C. Talocco, R. de Sire et al., "Intestinal permeability in physiological and pathological conditions: major determinants and assessment modalities," European Review for Medical and Pharmacological Sciences, vol. 23, no. 2, pp. 795-810, 2019.

[9] L. V. Hooper and A. J. Macpherson, "Immune adaptations that maintain homeostasis with the intestinal microbiota," Nature Reviews. Immunology, vol. 10, no. 3, pp. 159-169, 2010.

[10] W. Holzapfel, A. Arini, M. Aeschbacher, R. Coppolecchia, and B. Pot, "Enterococcus faecium SF68 as a model for efficacy and safety evaluation of pharmaceutical probiotics," Beneficial Microbes, vol. 9, no. 3, pp. 375-388, 2018.

[11] L. S. Celiberto, R. Bedani, N. N. Dejani et al., "Effect of a probiotic beverage consumption (enterococcus faecium CRL 183 and Bifidobacterium longum ATCC 15707) in rats with chemically induced colitis," PLoS One, vol. 12, no. 4, article ???, 2017.

[12] G. Sohail, X. Xu, M. C. Christman, and T. A. Tompkins, "Probiotic Medilac- $S((\mathrm{R}))$ for the induction of clinical remission in a Chinese population with ulcerative colitis: a systematic review and meta-analysis," World Journal of Clinical Cases, vol. 6, no. 15, pp. 961-984, 2018.

[13] J. Seishima, N. Iida, K. Kitamura et al., "Gut-derived Enterococcus faecium from ulcerative colitis patients promotes colitis in a genetically susceptible mouse host," Genome Biology, vol. 20, no. 1, p. 252, 2019.

[14] M. Sarra, F. Pallone, T. T. MacDonald, and G. Monteleone, "IL-23/IL-17 axis in IBD," Inflammatory Bowel Diseases, vol. 16, no. 10, pp. 1808-1813, 2010.

[15] S. Schreiber, T. Heinig, H. G. Thiele, and A. Raedler, "Immunoregulatory role of interleukin 10 in patients with inflammatory bowel disease," Gastroenterology, vol. 108, no. 5, pp. 1434-1444, 1995.

[16] E. C. Steinbach and S. E. Plevy, "The role of macrophages and dendritic cells in the initiation of inflammation in IBD," Inflammatory Bowel Diseases, vol. 20, no. 1, pp. 166-175, 2014.

[17] I. Schoultz, J. D. Soderholm, and D. M. McKay, "Is metabolic stress a common denominator in inflammatory bowel disease?," Inflammatory Bowel Diseases, vol. 17, no. 9, pp. 20082018, 2011.

[18] V. Eeckhaut, K. Machiels, C. Perrier et al., "Butyricicoccus pullicaecorum in inflammatory bowel disease," Gut, vol. 62, no. 12, pp. 1745-1752, 2013.

[19] D. N. Frank, C. E. Robertson, C. M. Hamm et al., "Disease phenotype and genotype are associated with shifts in intestinalassociated microbiota in inflammatory bowel diseases," Inflammatory Bowel Diseases, vol. 17, no. 1, pp. 179-184, 2011.

[20] H. Sokol, V. Leducq, H. Aschard et al., "Fungal microbiota dysbiosis in IBD," Gut, vol. 66, no. 6, pp. 1039-1048, 2017.

[21] K. Nones, B. Knoch, Y. E. Dommels et al., "Multidrug resistance gene deficient (mdrla-/-) mice have an altered caecal microbiota that precedes the onset of intestinal inflammation," Journal of Applied Microbiology, vol. 107, no. 2, pp. 557-566, 2009.
[22] M. Kummen, K. Holm, J. A. Anmarkrud et al., "The gut microbial profile in patients with primary sclerosing cholangitis is distinct from patients with ulcerative colitis without biliary disease and healthy controls," Gut, vol. 66, no. 4, pp. 611619, 2017.

[23] D. Gevers, S. Kugathasan, L. A. Denson et al., "The treatmentnaive microbiome in new-onset Crohn's disease," Cell Host \& Microbe, vol. 15, no. 3, pp. 382-392, 2014.

[24] S. A. Walujkar, S. V. Kumbhare, N. P. Marathe et al., "Molecular profiling of mucosal tissue associated microbiota in patients manifesting acute exacerbations and remission stage of ulcerative colitis," World Journal of Microbiology and Biotechnology, vol. 34, no. 6, p. ???, 2018.

[25] N. Kamada, Y. G. Kim, H. P. Sham et al., "Regulated virulence controls the ability of a pathogen to compete with the gut microbiota," Science, vol. 336, no. 6086, pp. 1325-1329, 2012.

[26] B. J. Meijer and L. A. Dieleman, "Probiotics in the treatment of human inflammatory bowel diseases: Update 2011," Journal of clinical gastroenterology, vol. 45, pp. S139-S144, 2011. 